\title{
O.S.P.
}

L'orientation scolaire et professionnelle

$30 / 3 \mid 2001$

Juger et être jugé : perspectives psychosociales

\section{Juger et estimer la valeur d'autrui : des biais de jugement aux compétences sociales}

Judging and estimating other people's value: from judgment biases to social competence

Éric Dépret et Laurence Filisetti

\section{CpenEdition}

Journals

Édition électronique

URL : http://journals.openedition.org/osp/5090

DOI : 10.4000/osp.5090

ISSN : 2104-3795

Éditeur

Institut national d'étude du travail et d'orientation professionnelle (INETOP)

Référence électronique

Éric Dépret et Laurence Filisetti, « Juger et estimer la valeur d'autrui : des biais de jugement aux compétences sociales », L'orientation scolaire et professionnelle [En ligne], 30/3 | 2001, mis en ligne le 15 septembre 2004, consulté le 16 décembre 2020. URL : http://journals.openedition.org/osp/5090 DOI : https://doi.org/10.4000/osp.5090

Ce document a été généré automatiquement le 16 décembre 2020.

(C) Tous droits réservés 


\section{Juger et estimer la valeur d'autrui : des biais de jugement aux compétences sociales}

Judging and estimating other people's value: from judgment biases to social competence

Éric Dépret et Laurence Filisetti

\section{Juger et estimer la valeur d'autrui}

1 Juger quelqu'un est un acte éminemment social à plus d'un titre. Non seulement la cible et la source du jugement sont engagées dans une relation interpersonnelle (et dans les processus cognitifs, affectifs et comportementaux qu'elle implique), mais elles sont également positionnées dans des rôles sociaux qui prescrivent leurs conduites respectives et le rapport social d'évaluation est lui-même inscrit dans un contexte social, organisationnel, et culturel dont les normes et les valeurs dirigent le processus d'évaluation.

On peut décliner la problématique générale du jugement social sous la forme de trois questions essentielles : juger quoi ? Pourquoi ? Comment ? En ce qui concerne l'objet du jugement, estime-t-on la valeur des comportements que l'on observe ou celle de la personne qui les émet et comment passe-t-on de l'un à l'autre ? En ce qui concerne l'objectif du jugement, évalue-t-on autrui dans le but de sanctionner, de prédire ou de modifier ses comportements futurs (voir, par exemple, la distinction que propose C. Hadji (1989) entre l'évaluation sommative, diagnostique et formative) ? En ce qui concerne la méthode du jugement, quelles mesures utilise-t-on (tests standardisés, observations cliniques), quelles comparaisons effectue-t-on (interpersonnelles, intrapersonnelles, passé/présent, attente/résultat, idéal/réel...) et sur quelles dimensions de valeurs socialement partagées, bref, quelles informations utilise-t-on 
(pertinentes, non pertinentes) et comment les intègre-t-on sous la forme d'un jugement?

3 Si le problème des méthodes de jugement pose directement la question de la validité des jugements émis, les trois dimensions suggérées (objet, objectif et méthode) interagissent entre elles de telle sorte que l'objectif du jugement, sa fonction sociale, conditionne souvent tant son objet que sa méthode. Dès lors, on ne doit pas s'étonner que le débat passionnel quant au rôle et à la fonction sociale du système scolaire (e.g. Bourdieu \& Passeron, 1970) soit pratiquement indissociable du débat sur les pratiques d'évaluation à l'école (pour une revue, voir e.g. Hadji, 1997; Lieury, 1996; Merle, 1998; Noizet \& Caverni, 1978). En effet, parce qu'elle est une institution de socialisation (d'intégration, de reproduction ?) par excellence, l'école est également une institution d'évaluation exemplaire. Il est incontestable qu'à travers ses fonctions d'instruction, d'éducation, d'orientation ou de sélection, la pratique de l'évaluation (des performances, des acquisitions, des compétences) est au coeur du dispositif scolaire qui estime la valeur des élèves et tend à les positionner dans la catégorie des «bons » ou des " mauvais ". Indiscutablement, " le système scolaire discrimine, hiérarchise, distingue, récompense et sélectionne » (Monteil, 1990, p. 80). C'est pourquoi l'étude des pratiques d'évaluation est un thème central en sciences de l'éducation. C'est également la raison pour laquelle les chercheurs en psychologie qui s'intéressent aux processus de jugement social utilisent souvent le contexte scolaire comme lieu d'observation privilégié. L'objectif de cet article est de tenter de résumer, de manière aussi synthétique et didactique que possible, les principales contributions des recherches de psychologie sociale au problème de l'évaluation et du jugement social afin de dégager des pistes de réflexion, si ce n'est d'action, pour le professionnel dans le cadre scolaire. Nous soulignerons, dans un premier temps, en quoi les recherches de psychologie sociale mettent en garde l'évaluateur contre les nombreux biais, cognitifs et affectifs, qui affectent son jugement. Nous suggèrerons, dans un second temps, qu'il est utile de prendre en compte non seulement la source mais également la cible du jugement et leur interaction ce qui nous amènera à considérer la notion de compétence sociale.

\section{Des biais de jugement}

4 La notion de biais de jugement, de distorsions systématiques dans le traitement des informations aboutissant à des jugements erronés, est au coeur des recherches en cognition sociale, un domaine qui se situe à l'intersection de la psychologie cognitive et de la psychologie sociale, et qui a pour objectif d'étudier les processus cognitifs impliqués dans les conduites sociales en général et dans la perception des personnes en particulier (pour une revue, voir Fiske \& Taylor, 1991 ; Leyens \& Beauvois, 1997). Deux constats essentiels fondent ces recherches. D'une part, les gens ont peu, ou pas d'accès direct à leurs propres processus mentaux (Nisbett \& Wilson, 1977). Étant donné que ces processus sont le plus souvent non-contrôlés, automatiques ou inconscients (Uleman \& Bargh, 1989), il faut distinguer la manière dont les gens " pensent " (les processus déterminants les faits de conscience et les comportements) de la manière dont les gens " pensent qu'ils pensent " (les rationalisations de sens-commun, c'est-à-dire socialement validées, que les gens sont capables de fournir pour expliquer leur propre fonctionnement). D'autre part, bien que les gens se montrent en général excessivement confiants et attachés à leurs propres jugements (Fisehoff et al., 1977), la manière dont 
ils les élaborent et les mettent à l'épreuve des faits s'écarte souvent des critères de la raison scientifique. Sans vouloir résumer l'ensemble des biais de jugement répertoriés, on peut en rappeler les éléments essentiels (pour plus de détail voir, DrozdaSenkowska, 1997; Fiske \& Taylor, 1991 ; Kahneman, Slovic, \& Tversky, 1982; Nisbett \& Ross, 1980).

\section{Des biais socio-cognitifs}

5 La formation d'un jugement, consiste à produire des inférences à partir d'informations, ce qui implique plusieurs étapes de traitement de l'information qui sont autant de sources de biais potentiels (Fiske \& Taylor, 1991, p. 358). Dès l'étape du recueil de l'information, toutes les informations nécessaires au jugement ne sont pas traitées dans la mesure où, guidés par leurs théories et préjugés, les gens ont tendance à privilégier les informations susceptibles de confirmer leurs attentes (biais de confirmation). De plus, les informations ambiguës sont interprétées dans le sens des attentes, et en l'absence de telles attentes, les premières informations reçues ont une influence prépondérante car elles guident le traitement ultérieur de l'information (effet de primauté). Par ailleurs, les gens ont tendance à baser leurs jugements sur des échantillons d'information peu représentatifs, voire à ignorer totalement l'information statistique au profit d'exemples frappants et extrêmes (biais d'échantillonnage). Ils éprouvent, en outre, les plus grandes difficultés à combiner l'information statistique et à évaluer les probabilités conditionnelles (négligence des lignes de base - ils sont d'ailleurs, en général, de piètres statisticiens comme en témoigne le succès des loteries et autres jeux de hasard, victimes de ce que les chercheurs appellent l'illusion de contrôle). Quand il s'agit, enfin, d'intégrer les informations pour aboutir à un jugement, les gens n'appliquent pas toujours les règles de décision, et en particulier la pondération des différentes informations, qu'ils pensent pourtant appliquer. D' autant plus que le stockage et le rappel des informations sont également sources de biais : la mémoire est sélective et reconstructive. Finalement, des informations et des indices contextuels non-pertinents, conscients ou inconscients, internes ou externes, affectent massivement les jugements émis (effet de contraste perceptif, effet de dilution des informations, effet d'amorçage d'un concept ou d'un souvenir, conditionnement classique, effet de l'humeur, effet de Halo).

6 Ces recherches mettent en évidence que, lorsque les gens s'engagent dans un processus de traitement de l'information visant à produire des inférences, leur jugement a de bonnes chances d'être biaisé. Les chercheurs soulignent en particulier les faiblesses du sens commun quand il s'agit d'inférer une relation de cause à effet (erreurs d'attribution causale) ou plus simplement d'estimer la covariation entre deux variables (corrélations illusoires): les gens ont tendance à voir des relations causales ou des relations tout court, là où il n'y en a pas. Qui plus est, les gens ne s'engagent souvent même pas dans ces processus complexes de traitement de l'information pour émettre leurs jugements, mais appliquent des principes simples, des raccourcis de raisonnement, encore appelés heuristiques de jugement. Ainsi, ils émettront un jugement en fonction de la facililité avec laquelle des exemples leurs viennent à l'esprit (heuristique de disponibilité) ou de la facilité avec laquelle ils peuvent s'imaginer le cours d'un évènement (heuristique de simulation), sur la base d'une ressemblance de surface entre une cible et une catégorie (heuristique de représentativité) ou sur la base d'un point de comparaison pas toujours pertinent (heuristique d'ancrage-ajustement). 
7 Ces biais de traitement de l'information dépassent par leur généralité, bien qu'ils s'y appliquent, le cadre du jugement des personnes. En ce qui concerne le jugement des personnes proprement dit, deux points essentiels se dégagent des recherches (voir Leyens, Yzerbyt, \& Schardron, 1996 ; Yzerbyt \& Schadron, 1996). Premièrement, les gens sont victimes de leurs stéréotypes et préjugés. À partir d'un minimum d'informations, ils sont prompts à assigner la cible d'un jugement à une ou plusieurs catégories. Dès lors, les stéréotypes, schémas ou représentations mentales, associés aux catégories vont guider et déformer les perceptions : les informations confirmatoires seront activement recherchées, les informations contradictoires seront négligées ou réinterprétées, et les informations manquantes seront reconstruites dans le sens du schéma. On comprend pourquoi les stéréotypes et les préjugés ont la vie dure et pèsent lourd dans la perception et le jugement des personnes. Deuxièmement, les gens ont tendance à inférer des caractéristiques internes stables (des attitudes, des traits de personnalité) pour expliquer les conduites sans toutefois disposer des informations qui sont nécessaires à de telles inférences (les informations relatives à la stabilité du comportement dans le temps et dans différentes situations ainsi qu'au comportement d'autres personnes placées dans la même situation ; Kelley, 1972). Ainsi, bien que la causalité d'un comportement soit théoriquement attribuable à des facteurs de type interne (" dispositionnels »), externes (" situationnels») et à leurs interactions, les gens ont tendance à s'écarter de l'objectivité scientifique en privilégiant les explications de type " interne » (surtout quand ils sont de culture occidentale et quand il s'agit d'expliquer les comportements d'autrui). Cette tendance abusive, et quasiautomatique, à associer un comportement à un trait sous-jacent censé l'expliquer (biais de correspondance) est également qualifiée d'erreur fondamentale d'attribution causale (Ross, 1977) dans la mesure où les gens sous-estiment, voire négligent entièrement, les poids des déterminants externes, situationnels et instables, dans leurs explications des comportements d'autrui. Se représenter et juger autrui en termes de traits de personnalité, et de croyances implicites relatives à la co-occurence de ces traits (les théories implicites de la personnalité), apparaît comme une caractéristique essentielle de la psychologie naïve et quotidienne (Beauvois, 19M, 1999; Leyens, 1983). Cependant, et paradoxalement, il semblerait que les professionnels de l'évaluation soient encore plus susceptibles que les novices d'utiliser leurs stéréotypes et de commettre l'erreur fondamentale dans la mesure où (a) ils pensent disposer de l'expertise et de la légitimité pour juger (la cible leur semble " jugeable »), (b) ils disposent souvent d'un temps limité pour émettre leur jugement (cette contrainte les amenant à interrompre leurs investigations et à " geler » leurs opinions prématurément) et enfin, et peut-être surtout, (c) l'activité sociale d'évaluation est elle-même facilitée et légitimée par l'inférence de dispositions internes, de tendances profondes et naturelles, que l'évaluateur se contenterait d'enregistrer objectivement en dehors de l'arbitraire du cadre et des critères sociaux du jugement.

8 Ainsi, le constat essentiel des recherches en cognition sociale nous semble consister en une mise en garde contre les nombreux biais de traitement de l'information qui se glissent dans la perception et le jugement des personnes et à une mise en cause de la valeur scientifique des jugements de valeur " personnologiques " qu'affectionne l'évaluateur quotidien, novice ou professionnel, quand il conclut au manque d'intelligence et à l'imbécillité d'un tel ou au courage et au sens des responsabilités d'un autre. 
Une telle mise en cause de la validité et de l'objectivité des jugements serait, somme toute, relativement triviale, si les jugements erronés n'avaient pas l'étonnante faculté de générer leur propre confirmation dans la réalité. En effet, et c'est essentiel, dans le cadre de relations humaines dynamiques les comportements mutuels s'adaptent aux attentes réciproques (Snyder, 1984). Ainsi, par exemple, dans le cadre d'un entretien clinique, l'évaluateur à qui l'on demande d'estimer le niveau d'introversion d'une personne, orientera ses questions dans le sens de la confirmation (ex : « vous arrive-t-il de rougir en public ?») ce qui amènera la personne en question, non seulement à être jugée mais à s'estimer elle-même et à se comporter effectivement de façon plus introvertie que si on avait demandé à l'évaluateur d'estimer son niveau d'extraversion (Snyder \& Swann, 1978b). De même, que dans le cadre d'un jeu compétitif, il suffit de faire croire au joueur que son partenaire est quelqu'un d'agressif pour qu'il se montre lui-même plus agressif et qu'en conséquence son partenaire le devienne également (Snyder \& Swann, 1978a) ou que, dans le cadre d'un entretien d'embauche, les préjugés du recruteur affectent ses comportements verbaux et non-verbaux qui, à leurs tour, affectent la performance du candidat dans le sens attendu (Word, Zanna, \& Cooper, 1974). Les biais de jugement peuvent donc être lourds de conséquences et constituer de véritables "prophéties auto-réalisatrices ". Ce phénomène est bien connu dans le cadre scolaire sous le nom d'effet Pygmalion : induire chez l'enseignant des attentes de réussite vis-à-vis de certains élèves (choisis au hasard) peut produire non seulement une amélioration des notes attribuées à ces élèves mais aussi une amélioration réelle de leurs performances (Rosenthal \& Jacobson, 1968). De nombreux facteurs sont susceptibles d'influencer les attentes de réussite, et donc les comportements et les jugements, des enseignants à l'égard d'un élève : les résultats d'un test de Q.I. ou l'avis d'un psychologue (comme c'était le cas dans les recherches de Rosenthal), mais aussi les performances antérieures de l'élève, celles de ses aînés, la classe sociale ou l'origine ethnique de ses parents, son sexe ou encore sa beauté physique (Dusek \& Gail, 1983). Ainsi, une performance ambiguë tendra à être interprétée comme un signe d'intelligence, ou de manque d'intelligence, en fonction de l'origine sociale de l'enfant (Darley \& Gross, 1983) et les notes d'un élève tendront à aller dans le sens de ses aînés s'ils ont fréquenté la même école (Dusek et al., 1983). Finalement, on ne s'étonnera pas qu'une même copie puisse être notée différemment en fonction du correcteur, de son état d'humeur, de l'ordre des copies, des performances antérieures de l'élève ou du niveau des autres élèves (voir, e.g. Huteau, 1996; Bressoux \& Pansu, ce numéro).

\section{Des biais socio-affectifs}

Nous avons jusqu'à présent abordé la question du jugement social sous l'angle des biais socio-cognitifs, c'est-à-dire des nombreuses distorsions dans le processus de traitement de l'information sociale qui sont susceptibles d'entraîner une méconnaissance et des jugements erronés à l'égard d'autrui. Les chercheurs en cognition sociale mettent en cause l'objectivité des jugements de valeur personnologiques qui nous sont quotidiens et soulignent que juger ne signifie pas connaître autrui (Yzerbyt \& Schadron, 1996) : la connaissance évaluative s'écarte à ce point de la connaissance descriptive qu'elle ne semble pas participer du même ordre de connaissance (voir Dubois \& Beauvois, ce numéro).

11 D'autres facteurs, qui ne sont pas d'ordre socio-cognitif, mais d'ordre socioaffectif, affectent massivement nos sentiments et nos jugements à l'égard d'autrui. Qualifier ces 
facteurs de biais est sans doute problématique dans la mesure où l'idée d'un critère de vérité, la possibilité de l'objectivité et donc de l'erreur, semble difficilement applicable au domaine des sentiments (ne dit-on pas que l'amour et la haine sont aveugles et que, comme les goûts et les couleurs, ils ne se discutent pas ?). Nous parlerons néanmoins de biais socio-affectifs dans la mesure où (a) les gens méconnaissent souvent, voire dénient au profit de rationalisations acceptables, les déterminants de leurs sentiments à l'égard d'autrui et (b) les affects qu'ils éprouvent sont susceptibles de contaminer et de distordre une bonne partie de leurs perceptions, raisonnements et jugements à propos d'autrui.

Bien entendu, ce serait une gageure que de vouloir répertorier l'ensemble des facteurs qui conditionnent les affects interpersonnels, d'autant plus, qu'à notre connaissance, il n'existe que très peu de revues exhaustives sur le sujet en psychologie sociale (voir cependant, Bersheid, 1985 ; Reis, 1995 ; Belin-Michinov \& Monteil, 2001) et que la plupart des études portant sur les facteurs de l'attraction interpersonnelle se trouvent dispersées dans les domaines de recherche traitant des attitudes, des émotions, des relations intragroupes, interpersonnelles ou intimes, du couple ou de l'amitié (voir, e.g., Dubé, 1994 ; Maisonneuve \& Lamy, 1993; Kelley et al., 1983). On peut néanmoins tenter d'en synthétiser les conclusions les plus marquantes.

13 Les facteurs qui affectent la nature des sentiments que l'on éprouve pour autrui renvoient, évidemment, à des aspects fondamentaux du fonctionnement du soi et des motivations qui y sont attachées. Ainsi, si l'on tend généralement à percevoir et juger autrui en comparaison avec soi-même (ce qui donne lieu à certains biais égocentrés ou effets d'auto-référence dans la perception d'autrui) il est clair que ce processus de comparaison n'est pas sans conséquence pour l'estime de soi et que des facteurs d'ordre motivationnel visant à protéger l'amour propre y jouent un grand rôle (on parle généralement de biais d'auto-complaisance : par exemple, les gens auront tendance à penser que leurs traits indésirables sont communs chez les autres - effet de faux consensus -, que leurs traits désirables sont rares - effet de fausse unicité -' qu'ils se conforment plus aux valeurs communes que les autres - effet Primus Inter Pares -, et finalement, ils s'estiment généralement comme étant meilleurs et supérieurs à la moyenne). Les effets de l'amour propre, du narcissisme primaire diraient nos collègues psychanalystes, sur les perceptions, les sentiments et les comportements sociaux sont certainement parmi les effets les mieux documentés de l'ensemble des recherches en psychologie sociale. Retenons, pour ce qui nous occupe ici, que les sentiments que nous éprouvons pour autrui sont liés, de plusieurs manières, aux sentiments que nous éprouvons pour nous-même (selon qu'autrui constitue une menace ou au contraire un appui pour l'amour propre, selon le degré de sécurité ou d'insécurité de cet amour propre).

On ne s'étonnera pas, dès lors, que le déterminant qui se dégage, en premier lieu, des recherches sur l'attraction et les attitudes interpersonnelles soit le degré de ressemblance entre la source et la cible (similitude des attitudes, opinions et comportements, y compris identifications et appartenances groupales communes). Que ceci résulte d'une tendance narcissique généralisée (j'aime ceux qui sont comme moi) ou de la satisfaction dérivée de la validation des attitudes propres (j'aime ceux qui sont d'accord avec moi), ce constat est consistant avec le fait que la flatterie, comme tout ce qui renforce l'amour propre, soit un instrument redoutable de séduction (j'aime ceux qui disent m'aimer). De même que, comme le montrent les recherches sociométriques, Fun des déterminants 
majeurs de l'attraction est de penser qu'elle est réciproque (j'aime ceux qui, je pense, m'aiment), bien que les gens ne soient pas toujours clairvoyants quant à la nature des sentiments d'autrui à leur égard.

Un second facteur qui conditionne l'attraction interpersonnelle concerne le degré de gratification et de satisfaction des besoins qui est associé à la cible. Dans le cadre de la théorie de l'échange social (Thibaut \& Kelley, 1959) on peut en effet concevoir les relations humaines comme des transactions continues d'utilités, de coûts et de bénéfices, entre partenaires interdépendants. Dans cette optique, l'affection ou la désaffection, est proportionnelle à l'évaluation subjective du rapport coût/bénéfice. En termes associationnismes, les sentiments éprouvés pour autrui reflètent la mesure dans laquelle autrui est associé à des plaisirs/ déplaisirs, à des récompenses ou à des punitions (Lott \& Lott, 1965), la mesure dans laquelle transitent par autrui des coûts ou des bénéfices importants pour soi. Une telle approche, en termes d'intérêt, d'échange d'utilité et de satisfaction des besoins a de quoi surprendre une conception idéaliste de l'amour, de l'amitié et des relations interpersonnelles. Elle est cependant consistante avec le fait que le contexte d'interdépendance (coopérative ou compétitive) et les conflits d'intérêts objectifs conditionnent les sentiments subjectifs interpersonnels. Elle est également consistante avec le fait qu'un élément essentiel de la dynamique des relations affectives (e.g. la satisfaction maritale) concerne la perception de l'équité des échanges entre les partenaires. On observe, en effet, une tendance frappante des gens à s'appareiller sur le " marché des sentiments » (ex : les gens tendent à s'associer à des partenaires de même niveau de beauté) comme si les gens évaluaient ce qu'ils pouvaient obtenir en fonction de ce qu'ils ont à offrir et adaptaient en conséquence leur niveau d'aspiration ; comme si la satisfaction, l'engagement et la rupture des relations affectives relevaient, en effet, d'un calcul subjectif de ce qui est à perdre et à gagner et d'une perception de l'équité des échanges de valeurs ou d'utilités (bien entendu dans ce calcul peuvent entrer en jeu toutes sortes d'utilités, en fonction des besoins, de leur niveau de satiété, de la rareté des ressources, de sorte que par exemple, la beauté puisse être compensée par le statut social, la " gentillesse " ou le " dévouement "...).

Le troisième facteur de l'attraction interpersonnelle, dont l'importance justifie qu'il soit généralement considéré à part entière par les chercheurs du domaine, concerne l'apparence physique de la cible ou le degré d'attirance physique éprouvé envers la cible. Il est probable que les représentations communes sous-estiment largement l'impact de la beauté physique dans les sentiments et jugements de valeurs interpersonnels, bien que les comportements effectifs ne semblent pas s'y tromper, si l'on en juge par le temps, l'argent et les efforts que les gens consacrent, toutes cultures confondues, à soigner et à modifier leur apparence corporelle - soins esthétiques, odeurs, parures et vêtements - qui constituent autant de signes forts de valeur, d'appartenance et de statut social et qui, dans la culture occidentale, constituent un formidable marché commercial. Bien entendu, les critères de la beauté sont éminemment variables d'une culture, d'une époque à une autre, bien que l'on puisse probablement en repérer certains invariants d'ordre sociobiologique (e.g. le caractère juvénile des traits du visage, l'état de la dentition, la musculature chez l'homme, la taille des hanches chez la femme, qui signalent l'état de santé et les capacités de reproduction).

17 Un quatrième ensemble de facteurs, enfin, fait l'unanimité chez les chercheurs qui étudient l'attraction interpersonnelle. Il s'agit du degré de proximité, de familiarité et 
d'intimité entre la source et la cible. Proximité fonctionnelle, d'abord, celle qui permet, favorise ou entrave, la fréquence des interactions, des rencontres, des communications. Il y a là des prédicteurs environnementaux très puissants du développement ou de l'affaiblissement, des sympathies mutuelles (quant à la proximité physique proprement dite les évidences sont ambiguës: d'une part, les gens tendent à réagir négativement à la violation de leur espace personnel, les distances d'interaction appropriées, variables selon les cultures, constituant des normes puissantes ; d'autre part, il semble que le fait de toucher son interlocuteur puisse engendrer la sympathie) ${ }^{1}$. Familiarité, ensuite, dans la mesure où la fréquence des contacts semble être, à elle seule (toutes choses étant égales par ailleurs) un facteur de sympathie (effet dit de simple exposition). Intimité, enfin, dans la mesure où la révélation de soi, le partage social d'attitudes, d'émotions ou de sentiments intimes (qu'il est normalement inapproprié d'exprimer publiquement) engendrent la révélation réciproque et la sympathie.

Ressemblance, gratification narcissique et perception de réciprocité ; satisfaction des besoins, utilité, rapport coût/bénéfice et perception d'équité ; apparence et attirance physique ; proximité, familiarité et intimité ; tels sont, selon les recherches empiriques, les déterminants majeurs des attitudes interpersonnelles. On peut ajouter à cette liste deux autres facteurs essentiels qui émanent de la littérature classique en psychologie sociale.

D'une part, il ne suffit pas de considérer la valeur que représentent les attributs et les comportements de la cible à l'égard de la source mais également celle des comportements de la source à l'égard de la cible. En effet, selon la théorie de l'engagement (Kiesler, 1971) un comportement est d'autant plus engageant qu'il est émis librement, publiquement et qu'il est coûteux. Or, par souci de cohérence cognitive ou de réduction de leurs sentiments de dissonance, les gens ont tendance à modifier leurs attitudes dans le sens de leurs comportements engageants, à rationaliser leurs engagements (Beauvois \& Joule, 1981). Il s'ensuit que, la sympathie éprouvée pour la cible peut résulter des comportements engageants de la source à son égard (« si je lui ai tant donné c'est parce qu'elle le mérite, parce qu'elle m'est sympathique »). Machiavel ne s'y trompait pas quand il conseillait au prince, « les gens vous aimeront plus pour ce qu'ils ont fait pour vous que pour ce que vous avez fait pour eux ».

D'autre part, l'une des caractéristiques fondamentales des comportements sociaux est qu'ils sont régis par des normes, des standards partagés, propres à un groupe social, et relatifs aux attitudes et aux comportements considérés comme corrects, appropriés, désirables. Il s'ensuit que la normativité des attitudes et des conduites, leur adéquation avec les normes sociales en vigueur dans un groupe ou une société donnée, est un facteur essentiel d'approbation ou de désapprobation sociale. En ce sens, un leader populaire est normatif, il représente l'identité, les normes et les valeurs du groupe, alors qu'un membre déviant est impopulaire, rejeté. On peut, en fait, considérer que les normes sociales renvoient à des valeurs, des utilités sociales, dans un arbitraire social donné, et que leur apprentissage au cours de la socialisation, leur intériorisation et leur implémentation, constituent l'une des sources essentielles des jugements de valeur sociale que nous portons sur autrui.

21 Enfin, tout un ensemble de facteurs contextuels peut affecter de manière non négligeable nos attitudes à l'égard d'autrui. Une activation physiologique, même incidente, l'état d'humeur dans laquelle on se trouve ainsi que les éléments de l'environnement présents pendant l'interaction (autour d'un verre dans un restaurant 
agréable...) peuvent amener, par association ou erreur d'attribution, à trouver une personne plutôt sympathique ou plutôt antipathique. L'expérience d'un sort commun ou mieux, d'une menace commune, est connue pour engendrer de l'attraction interpersonnelle, d'autant que l'anxiété produit des tendances affiliatives et des comportements d'attachement envers les personnes qui remplissent alors une fonction de support social. Les éléments de comparaison et d'alternatives disponibles dans l'univers relationnel entrent également en jeu (« je t'aime moins parce que je le préfère »). Enfin, les recherches montrent qu'un sort, favorable ou défavorable, même s'il est objectivement incontrôlable et clairement indépendant de la volonté de celui qu'il affecte, engendre des réactions de sympathie ou d'antipathie dans l'entourage social de sorte que les gens semblent adhérer à une croyance en un Inonde juste dans lequel les gens obtiennent ce qu'ils méritent. Cette tendance à croire que la valeur des événements qui affectent les gens reflète leur valeur intrinsèque (les événements positifs arrivent aux gens biens, les événements négatifs arrivent à ceux qui le méritent), connue pour engendrer une double stigmatisation des victimes, est à rapprocher de l'erreur fondamentale d'attribution causale. Erreur d'attribution causale concernant, non pas les comportements, comme nous l'avons déjà vu (" les comportements d'une personne reflètent essentiellement ses caractéristiques internes, sa personnalité »), mais les renforcements (« la destinée et la condition d'une personne reflètent essentiellement ses caractéristiques internes, sa valeur »- et tout est pour le mieux dans le meilleur des mondes !).

Rappelons que décrire, comme nous l'avons fait, l'ensemble de ces facteurs sous la rubrique des "biais socio-affectifs " ne signifie pas que les sentiments que nous éprouvons à l'égard des autres sont basés sur de « mauvaises " raisons (quelles seraient les «bonnes » raisons ?). Cela signifie, simplement, que (a) les gens n'ont pas nécessairement conscience des déterminants de leurs propres affects et que les explications qu'ils en fournissent n'en constituent parfois que des rationalisations acceptables et que (b) parce que les systèmes affectifs et cognitifs sont loin d'être étanches (les psychologues sociaux les associent d'ailleurs sous le concept d'attitude), les sentiments que l'on éprouve envers une personne sont susceptibles de colorer l'ensemble des perceptions, des comportements et des jugements que l'on émet à son égard, comme le suggère le fait que les traits de personnalité que nous utilisons pour décrire les gens sont, non seulement, saturés de valeur mais également fortement corrélés entre eux sur le plan évaluatif : une dimension évaluative générale (positive/ négative, sympathie/antipathie) semble structurer les descriptions personnologiques (voir cependant Beauvois \& Dubois, ce numéro) ${ }^{2}$.

Qui prétendrait que, dans le cadre des relations maître-élève, les sentiments qu'éprouve le maître à l'égard de l'élève (du « chou-chou ", de «l'insupportable » voire de la "tête-à-claque ») n'affectent aucunement ses comportements, ses évaluations, son jugement ? Sans prendre le temps d'illustrer l'ensemble des biais socio-affectifs et leurs réalisations possibles dans le cadre scolaire, il suffit de savoir que l'on a tendance à punir plus durement un enfant (ou un accusé) au physique désagréable (dans les recherches de laboratoire comme dans les jurys d'assises) ! Il paraît donc légitime d'alerter l'évaluateur quotidien contre les biais, non seulement socio-cognitifs, mais socio-affectifs qui affectent son jugement et ses comportements. Car bien entendu, là comme ailleurs, se développent des cercles vicieux ou vertueux, de confirmation comportementale, de sorte que la sympathie engendre la sympathie ou qu'un 
sentiment d'antipathie engendre des comportements d'antipathie auxquels s'adapte en retour la cible par des sentiments et des comportements antipathiques.

\section{Que faire face aux biais ?}

24 À ce point de notre exposé, l'évaluateur quotidien, novice ou professionnel, commencet-il, peut-être, à douter de la validité, et de la nature des déterminants effectifs, de ses jugements. Dans quelle mesure mon jugement est-il basé sur un ensemble d'informations pertinentes, nécessaires et suffisantes ou sur une somme disparate d'informations partielles et partiales, déformées par mes préjugés et mes sentiments à l'égard de celui que j'évalue ? Dans quelle mesure les processus et les déterminants socio-cognitifs et socio-affectifs de mon jugement sont-ils connus de moi, contrôlables et explicitables ou plutôt inconscients, injustifiables voire inavouables ? Dans quelle mesure, enfin, les comportements de celui que j'évalue sont-ils le reflet de ses qualités propres, que je suis en droit de juger ou de mes propres préjugés tels qu'ils s'autoréalisent dans l'interaction évaluateur-évalué ? Bref, dans quelle mesure mon jugement est-il juste?

Ces questions dérangeantes amènent probablement le lecteur à se demander : que faire, face aux biais ? Les chercheurs du domaine s'accordent certainement sur deux points. D'une part, la plus grande prudence doit être de mise dans le domaine de l'évaluation et, tout en se méfiant des jugements hâtifs, il est souhaitable d'adopter des procédures de standardisation et de contrôle susceptibles de limiter l'influence des biais (e.g. des tests fiables, valides, anonymes...). D'autre part, il est souhaitable de contribuer à l'information et à la prise de conscience des déterminants cognitifs et affectifs connus du jugement social (c'est le sens de cet article) car, tout en étant l'objet de déterminations, il n'en reste pas moins que la capacité d'auto-réflexion, et donc d'autocréation, constitue l'essence du sujet (du projet ?) humain (" connais-toi toi-même... »). Par-delà ce constat, les avis divergent selon les écoles de pensée. Pour les uns, le sujetjuge est un scientifique en herbe, dans la mesure où il cherche à s'approcher de la connaissance objective. Ses erreurs de jugement sont liées à des difficultés spécifiques de raisonnement. Il faut donc apprendre aux gens à raisonner, projet qui n'est pas simple dans la mesure où il ne suffit pas que les gens comprennent les principes du raisonnement, encore faut-il qu'ils en perçoivent la pertinence et les appliquent dans des situations spécifiques. D'autant que, pour d'autres, le sujet-juge est avant tout un avare cognitif. Ses capacités de traitement de l'information sont limitées et face à la complexité de l'environnement il est condamné, pour s'adapter, à simplifier les données et à utiliser des raccourcis de jugement, peu coûteux, mais sources d'erreur. Une troisième optique, plus optimiste, considère le sujet-juge comme un tacticien motivé, dans la mesure où un ensemble des facteurs d'ordre motivationnel peuvent amener les gens à suspendre leur jugement et à mobiliser leurs ressources cognitives pour passer d'un traitement superficiel et heuristique de l'information à un traitement plus approfondi et systématique. Quoiqu'il en soit de ses espoirs d'amélioration, qu'il soit considéré comme un scientifique en herbe, un avare cognitif ou un tacticien motivé, c'est bien sur les défauts et les manques du sujet qui juge (manque de connaissance, manque de capacité, manque de motivation) qu'insistent la plupart des chercheurs en cognition sociale. Or, comme nous l'avons déjà souligné, s'il est légitime de reprocher à celui qui juge de ne pas traiter correctement (rationnellement, objectivement) l'information dont il dispose, il semble plus difficile de lui reprocher d'éprouver des 
sentiments à l'égard de la cible, et de les mettre en action dans l'interaction comportementale, c'est-à-dire d'être autre chose qu'une machine à traiter de l'information. Une approche complémentaire nous semble donc envisageable qui prenne en compte non seulement les compétences de celui qui juge, mais également celles de celui qui est jugé. Dans cette optique, la question du jugement social n'est plus seulement : quelle est la nature des processus propres à la source qui affectent son jugement (et comment les améliorer) ? Mais également : quels sont les processus propres à la cible qui affectent le jugement de la source ? Et finalement: comment contribuer à l'amélioration de la relation dynamique entre l'évaluateur et l'évalué en considérant simultanément les deux pôles de l'interaction?

\section{Des compétences sociales}

La notion de compétence sociale est une notion assez vaste, dont la polysémie est d'ailleurs renforcée par l'emploi indifférencié du singulier (la compétence sociale) ou du pluriel (les compétences sociales) par les auteurs qui s'y réfèrent. Dans son acception la plus générale la compétence sociale dénote un certain savoir-faire social : il s'agit de la capacité, dans un contexte social donné, à interagir avec autrui de manière socialement efficace, à adopter des comportements socialement adaptés et à entretenir des relations sociales satisfaisantes. L'importance de ce savoir-faire social était déjà soulignée par Thorndike (1920), l'un des pères de la psychométrie, qui distinguait l'intelligence sociale, définie comme la capacité à agir judicieusement dans les relations humaines, des autres formes d'intelligence. Plus récemment, la compétence sociale a été définie comme la capacité à accomplir des tâches interpersonnelles (Weinstein, 1969), à accomplir des objectifs sociaux dans un contexte social spécifique (Ford, 1982), à décoder correctement l'information sociale afin d'y adapter en conséquence son comportement (Walker \& Foley, 1973), à se comporter de manière adaptée, efficace, socialement acceptable ou estimée comme telle, dans une situation donnée (Combs \& Slaby, 1977), à adopter des comportements sociaux qui soient positivement renforcés par l'entourage social et à éviter ceux qui sont réprimés ou punis (Libet \& Lewinshom, 1973).

Selon la littérature du domaine (pour une revue, voir Ford \& Tisak, 1983) il semble que deux facteurs principaux sous-tendent ce savoir-faire social que l'on nomme compétence sociale. D'une part, le degré de compréhension de l'autre, du sens de l'interaction et de l'évènement social, qui implique le décodage et l'analyse de l'information sociale (notamment des indices non-verbaux ; Walker \& Foley, 1973), l'empathie et la sensibilité sociale (Feshbach \& Feshbach, 1987; Ford, 1982), ce que certains auteurs résument sous le terme de " conscience sociale " : la compréhension des causes et des significations du comportement d'autrui, y compris des sentiments et des émotions qui le sous-tende (Greenspan, 1981). D'autre part, le degré d'adhésion ou de respect des règles, normes et rôles sociaux en vigueur dans un groupe social donné que certains auteurs qualifient de " responsabilité sociale " (Wentzel, 1991a). L'importance des normes sociales en tant que régulateurs des comportements sociaux et déterminants du jugement social n'est, en effet, plus à démontrer. Un troisième facteur, plus marginalement mentionné dans la modélisation des compétences sociales, concerne le degré de retenue et de contrôle de soi (Feldman \& Wentzel, 1990) étant donné qu'un 
comportement adapté ne peut être produit qu'avec calme et réflexion (Greenspan, 1981).

Sur le plan empirique, l'opérationnalisation des compétences sociales pose problème : comment mesurer, à partir de quels indices inférer, le niveau de compétence social ? Étant donné que la notion de compétence sociale n'a de sens qu'en fonction des conséquences du comportement social dans un contexte social spécifique, de son efficacité, certains auteurs plaident pour une opérationnalisation en termes d'atteinte de but : amener quelqu'un à faire quelque chose ou à accepter une requête, communiquer efficacement ou persuader (e.g. Ford et al., 1983). Les stratégies comportementales utilisées dans l'interaction pour atteindre ces buts peuvent être mesurées, en situation naturelle ou en laboratoire, à l'aide de grilles d'observation et d'enregistrements audiovisuels (e.g. Gottman et al., 1973 ; Green et al., 1980; Ford et al., 1983). D'autres auteurs insistent plutôt sur les capacités d'analyse et de compréhension des situations d'interaction sociale, la mesure des compétences sociales prenant alors la forme de tests psychométriques où il s'agit de prédire correctement les comportements d'un acteur, de décoder ses émotions ou de résoudre des dilemmes sociaux (que feraistu ou que devrait faire l'acteur, dans cette situation? voir, e.g. Keating, 1978). Enfin, la majorité des chercheurs évaluent les compétences sociales à partir des jugements de l'entourage social, soit sous la forme de mesures sociométriques classiques (expressions d'affection ou de rejet, choix de partenaire, mesures de popularité) ou d'échelles plus spécifiques (qui dans le groupe coopère le plus, est bon camarade, menace les autres ou initie les conflits ; voir, e.g. Feldman et al., 1990; Gottman et al., 1975; Green et al., 1980; Wentzel, 1991b, 1993; Wentzel \& Cadwell, 1997). Ainsi, dans la plupart des recherches, la compétence sociale est inférée à partir des jugements de sympathie ou d'approbation sociale : conçue comme la capacité à engendrer des jugements favorables, la question des compétences sociales correspond bien, en miroir, à celle du jugement social selon que l'on aborde la question prioritairement sous l'angle de la source ou sous l'angle de la cible du jugement.

Sur le plan scolaire, de nombreuses recherches ont mis en évidence un lien étroit entre la compétence sociale et la réussite scolaire des élèves : un enfant populaire, qui sait se faire apprécier de ses pairs et qui possède de bonnes compétences relationnelles, réussit mieux à l'école qu'un enfant impopulaire (Feldman et aL, 1990; Feschbach et al., 1987; Green et al., 1980; Wentzel, 1991b, 1993, 1998 ; Wentzel et al., 1997). Bien entendu, le lecteur averti interprètera ces recherches corrélationnelles avec prudence (il est possible, en effet, que ce soit la réussite scolaire qui influence la popularité et non l'inverse ou encore que performance scolaire et compétence sociale soient influencées conjointement par un troisième facteur). Les analyses statistiques de régression suggèrent, néanmoins, l'existence d'une relation causale entre compétence sociale et performance scolaire (Felman et al., 1990 ; Feschbach et al., 1987 ; Wentzel, 1991b, 1993, 1998 ; Luthar, 1995). Selon les chercheurs, cette relation serait médiatisée par plusieurs facteurs (Wentzel, 1991a, 1998 ; Gottman et al., 1975; Vosk et al., 1982; Wentzel et al., 1995, 1997). D'une part, le fait de savoir interagir avec les autres et d'être apprécié de ses camarades et de ses enseignants induit un confort émotionnel et, exempt de préoccupations relationnelles, l'élève peut investir toutes ses ressources dans les activités scolaires. Inversement, un élève qui est rejeté par ses camarades et ses enseignants éprouve un mal-être affectif qui peut s'actualiser sous la forme d'anxiété, d'auto-dépréciation, de baisse de motivation pour les activités scolaires, voire de rejet des situations scolaires en général. D'autre part, un élève qui se sent à l'aise avec son 
entourage n'hésite pas à coopérer, à demander et à recevoir de l'aide, ce qui favorise les apprentissages. Inversement, un élève qui éprouve des difficultés relationnelles recevra moins d'aide et de soutien.

$\mathrm{Au}$ vu de ces considérations, tant à propos de l'importance des compétences sociales à l'école et de leur effet sur les performances scolaires que des biais socio-cognitifs et socio-affectifs qui affectent le jugement et les comportements des enseignants, nous voulons suggérer que la compétence sociale d'un élève dans ses interactions avec son maître est un facteur important de son adaptation et de sa réussite scolaire. En d'autres termes, que l'on considère la compétence sociale au sens général comme savoir-faire social et capacité relationnelle ou au sens plus spécifique comme capacité à faire bonne impression, à susciter approbation sociale et affection, la compétence sociale de la cible dans sa relation à la source du jugement est un facteur déterminant du jugement social. L'impression favorable que celui qui est évalué saura susciter et la relation satisfaisante qu'il saura entretenir avec celui qui l'évalue est susceptible d'affecter l'ensemble des dimensions du jugement et surtout d'engendrer chez l'évaluateur des comportements favorisant l'épanouissement des performances de celui qu'il juge, dans le cadre scolaire comme ailleurs.

31 Par ailleurs, tant au vu des recherches sur le jugement social que sur la compétence sociale il est clair que la normativité des conduites est un facteur essentiel du jugement et que la connaissance et l'implémentation des normes sociales en vigueur dans un groupe est une dimension essentielle de la compétence sociale. Plus précisément, nous voulons souligner l'importance des normes sociales relatives aux comportements d'interaction sociale. Il existe en effet dans toutes les cultures des normes fondamentales concernant la manière correcte, appropriée, désirable d'interagir avec autrui (que dire, que faire quand on rencontre quelqu'un - un inconnu, un ami, un supérieur hiérarchique -? Comment formuler une requête ?). Ces normes de l'interaction sont certainement un objet privilégié du processus de socialisation et sont apprises très tôt car d'elles dépend d'abord l'adaptation sociale (" dis bonjour, au revoir à la dame »; " dis s'il vous plait, merci, excusez-moi »...). Dans notre culture, on fait couramment référence à ces normes sous le terme de "politesse " (pour une discussion, voir Holtgraves, 1992). Ne dit-on pas d'un enfant " poli » ou " impoli » que c'est essentiellement un enfant "bien » ou " mal élevé »? Dans l'une de nos recherches (Filisetti \& Dépret, 2000), nous avons demandé à 70 enfants de classe primaire (cycle III) de formuler une requête à un enseignant inconnu, dans le cadre d'un jeu de rôle, selon trois consignes : « comme tu le ferais en vrai », « comme le ferait un enfant impoli », « comme le ferait un enfant poli ». Les enregistrements vidéos des 210 interactions ainsi recueillies ont ensuite été soumis aux jugements d'un panel de 60 enseignants d'écoles primaires à qui l'on demandait d'évaluer chaque enfant sur trois critères : (a) la valeur scolaire (" dans quelle mesure diriez-vous de cet enfant qu'il est un bon/mauvais élève »), (b) la sympathie (« dans quelle mesure diriez-vous de cet enfant qu'il vous semble sympathique/antipathique »), (c) la politesse ("dans quelle mesure diriez-vous de cet enfant qu'il est poli/impoli »). Outre le fait que l'analyse qualitative et quantitative des enregistrements vidéos nous a permis de spécifier la manière dont ces enfants se représentent et implémentent la politesse (essentiellement les expressions de respect de la valeur d'autrui et de la liberté d'autrui, et dans une moindre mesure, le contrôle moteur ; voir Filisetti \& Dépret, 2000), deux constats importants sont à retenir pour ce qui nous occupe ici : d'une part, les comportements de politesse ou d'impolitesse des élèves affectent massivement le jugement des enseignants, non seulement la sympathie 
éprouvée à leur égard mais aussi l'évaluation de leur valeur scolaire. D'autre part, bien que la plupart des élèves aient une représentation bien différenciée de ce qu'est la politesse et l'impolitesse, ce n'est pas le cas pour tous les élèves : tous ne semblent pas être " clairvoyants " à l'égard de cette norme. Dès lors se pose la question des différences socio-culturelles qui peuvent constituer de véritables handicaps dans l'interaction sociale et, plus généralement, la question de la formation des compétences sociales à l'école (voir Oden \& Asher, 1977).

\section{Conclusion}

La question du jugement social, de l'évaluation et des jugements de valeur que nous portons sur autrui, est une question essentielle et complexe qui est au cœur des préoccupations de la psychologie sociale. De nombreux biais d'ordre socio-cognitifs et sociaux-affectifs altèrent les jugements et, outre les conseils de prudence et de rigueur dans l'évaluation des personnes qui émanent des chercheurs, il est bon pour l'évaluateur quotidien, naïf ou professionnel, de mieux connaitre et de prendre conscience des facteurs qui affectent ses jugements. Il nous semble particulièrement important de prendre conscience de l'aspect dynamique, interactif, confirmatoire et auto-réalisateur de la relation d'évaluation et de considérer le rapport d'évaluation comme une relation humaine, dans le cadre scolaire comme ailleurs. Indiscutablement, des facteurs d'ordre non seulement cognitifs mais aussi affectifs se jouent dans la relation maitre-élève et en déterminent les conséquences tant au plan de l'évaluation que de la performance effective.

Dès lors que l'on considère l'évaluation comme une relation d'évaluation, on est amené à s'interroger sur les deux pôles de la relation, non seulement sur les facteurs propres à la source mais aussi sur les facteurs propres à la cible du jugement. Bien que la majorité des chercheurs en psychologie sociale se soient focalisés sur la source du jugement (les recherches sur les biais du jugement social) il nous semble nécessaire de considérer également la cible du jugement (les recherches sur les compétences sociales). La notion de compétence sociale nous semble, en effet, particulièrement utile et heuristique, pour peu que celle-ci ne soit pas réifiée (considérée comme une chose naturelle existant en soi). En effet, la notion de compétence sociale n'a de sens que dans le cadre d'un arbitraire social donné (e.g. la connaissance et l'intégration des normes sociales en vigueur dans un groupe).

L'intérêt d'une approche du jugement social et de l'évaluation en termes de compétence sociale est double. D'une part, sur le plan théorique, l'étude des compétences sociales ouvre des voies de recherche prometteuses pour la compréhension du comportement social en général. On peut se demander, par exemple, comment s'agencent la genèse et le développement des compétences sociales, sachant que l'on peut supposer que, dans un groupe social donné, l'ordonnancement des compétences sociales correspond aux aspects les plus fondamentaux du fonctionnement $\mathrm{du}$ groupe et des comportements sociaux. On peut également envisager d'autres enrichissements mutuels entre psychologie sociale et psychologie du développement dans la mesure où l'on peut considérer l'ensemble des stratégies connues d'influence, d'auto-présentation et de gestion de l'impression sous l'angle des compétences sociales et de leur développement. D'autre part, sur le plan appliqué, l'étude des compétences sociales ouvre la voie vers une meilleure prise en compte des 
différences interpersonnelles de savoir-faire social, dans un univers social donné, et pose le problème de la formation des compétences sociales. À quand une école qui reconnaisse de plein droit l'importance des compétences sociales, leur impact, de fait, dans l'évaluation et la relation éducative, et la nécessité de doter les élèves non seulement d'outils intellectuels mais également d'outils relationnels leur permettant de s'adapter et d'intégrer l'univers social?

\section{BIBLIOGRAPHIE}

Beauvois, J.-L. (1984). La psychologie quotidienne. Paris : P.U.F.

Beauvois, J.-L. (1999). Psychologie quotidienne. In H. Bloch, R. Chemana, E. Dépret, A. Gallo, P. Leconte, J.-F. Le Ny, J. Postel, \& M. Reuchlin (Éds.), Grand dictionnaire de la psychologie (pp. 738-740). Paris : Larousse.

Beauvois, J.-L., \& Joule, R. (1981). Soumission et idéologies. Pans: P.U.F.

Belin-Michinov, E., \& Monteil, J.-M. (2001). Attraction et relations interpersonnelles. Approches scientifiques et repères pour l'action. In J.-M. Monteil \& J.-L. Beauvois (Éds.), La psychologie sociale.' Des compétences pour l'application (Vol. 5). Grenoble : P.U.G.

Berscheid, E. (1985). Interpersonal attraction. In G. Lindzey \& E. Aronson (Eds.), The Handbook of Social Psychology (3' édition) (pp. 413-484). New York : Lawrence Erlbaum Associates. Bourdieu, P., \& Passeron, J.C. (1970). La reproduction. Eléments pour une théorie du système d'enseignement. Paris : Les éditions de minuit.

Combs, M. L., \& Slaby, D. A. (1977). Social skills training with children. In B. B. Lahey \& A. E. Kazdin (Eds.), Advances in Clinical Child Psychology, Vol 1. New-York : Plenum Press.

Darley, J. M., \& Gross, P. H. (1983). A hypothesis-confirming bias in labeling effects. Journal of Personality and Social Psychology, 44, 20-33.

Drozda-Senkowska, E. (1997). Les pièges du raisonnement. Paris : Retz.

Dubé, L. (1994). Les relations interpersonnelles. In R. J. Vallerand (Éd.), Les Fondements de la Psychologie Sociale (pp. 457-502). Montréal: Gaétan Morin.

Dusek, J. B., \& Gai!, J. (1983). The bases of teacher expectancies : A meta-analysis. Journal of Educational Psychology, 7, 3, 327-346.

Feldman, S. S., \& Wentzel, K. R. (1990). Relations among family interaction patterns, classroom self-restraint, and academie achievement in preadolescent boys. Journal of Educational Psychology, $82,4,813-819$.

Feshbach, N. D., \& Feshbach, S. (1987). Affective processes and academie achievement. Child Development, 58, 1335-1347.

Filisetti, L., \& Dépret, É. (2000). La politesse comme compétence sociale : Son impact sur les jugements des enseignants, sa représentation par les élèves. Manuscrit non publié. Université Pierre Mendes France, Grenoble. 
Fischoff, B., Slovic, P., \& Lichtenstein, S. (1977). Knowing with certainty : The appropriateness of extreme confidence. Journal of Experimental Psychology : Human Perception and Performance, 3 , 552-564.

Fiske, S. T., \& Taylor, S. E. (1991). Social Cognition. New York : McGraw-Hill.

Ford, M. E. (1982). Social cognition and social competence in adolescence. Developmental Psychology, 18, 3, 323-340.

Ford, M. E., \& Tisak, M. T. (1983). A further search for social intelligence. Journal of Educational Psychology, 75, 2, 196-206,

Gottman, J., Gonso, J., \& Rasmussen, B. (1975). Social interaction, social competence, and friendship in children. Child Development, 46, 709-718.

Green, K. D., Forehand, R., Beek, S. J., \& Vosk, B. (1980). An assessment of the relationship among measures of children's social competence and children's academie achievement. Child Development, 51, 1149-1156.

Greenspan, S. (1981). Defining childhood social competence : A proposed working model. Advances in Special Education, 3, 1-39.

Hadji, C. (1989). L'évaluation, règles du jeu. Paris : E.S.F.

Hadji, C. (1997). L'évaluation démystifiée. Paris : E.S.F.

Holtgraves, T. (1992). The linguistic realization of face management : Implications for language production and comprehension, person perception, and cross-cultural communication. Social Psychology Quaterly, 55, 2, 141-159.

Huteau, M. (1996). L'évaluation par les notes et par les tests. In A. Lieury (Éd.), Manuel de psychologie de l'éducation et de la formation (pp. 271-302). Pans: Dunod.

Kahneman, D., Slovic, P., \& Tversky, A. (1982). Judgement under uncertainty Heuristics and biases. New York : Cambridge University Press.

Keating, D. P. (1978). A search for social intelligence. Journal of Educational Psychology, 70, 2, 218-223.

Kelley, H. H. (1972). The process of causal attribution. American Psychologist, 28, 107-128. Kelley, H. H., Berscheid, A. C., Harvey, J. H., Huston, T. L., Levinger, G., McClintock, E., Peplau,

L. A., \& Peterson, D. R. (1983). Close relationships. New York : Freeman and Company. Kiesler, C. A. (1971). The psychology of commitrnent. Experiments linking behavior to belief

New York : Academie Press.

Leyens, J.-P. (1983). Sommes-nous tous des psychologues ? Bruxelles : Mardaga.

Leyens, J.-P., \& Beauvois, J.-L. (1997). La psychologie sociale : L'ère de la cognition (Vol. 3). Grenoble : P.U.G.

Leyens, J.-P., Yzerbyt, V., \& Schadron, G. (1996). Stéréotypes et cognition sociale. Bruxelles : Mardaga.

Libet, 1., \& Lewinshom, P. M. (1973). The concept of social skill with special references to the behavior of depressed persons. Journal of Consulting and Clinicat Psychology, 40, 304-312.

Lieury, A. (1996). Manuel de psychologie de l'éducation et de la formation. Paris : Dunod. Lott, A. J., \& Lott, B. E. (1965). Group cohesiveness as interpersonal attraction : A review of relationships with antecedent and consequent variables. Psychological Bulletin, 64, 259-309. 
Maisonneuve, J., \& Lamy, L. (1993). Psycho-sociologie de l'amitié. Paris : P.U.F.

Merle, P. (1998). Sociologie de l'évaluation scolaire. Que sais-je, 3278. Paris : P.U.F.

Monteil, J.-M. (1990). Eduquer et former : Perspectives psycho-sociales. Grenoble : P.U.G.

Nisbett, R. E., \& Ross, L. (1980). Human inference Strategies and shortcomings of social judgment. Englewood Cliffs, New Jersey : Prentice Hall.

Nisbett, R. E., \& Wilson, T. D. (1977). Telling more than we can know : Verbal reports on mental processes. Psychological Review, 84, 231-259.

Noizet, G., \& Cavemi, J.-P. (1978). Psychologie de l'évaluation scolaire. Paris : P.U.F.

Oden, S., \& Asher, S. R. (1977). Coaching children in social skills for friendship making. Child Development, 48, 495-506.

Reis, H. T. (1995). Attraction. In A. Manstead, \& M. Hewstone (Eds.), The blackwell encyclopedia of social psychology (pp. 57-61). Oxford : Blackwell.

Rosenthal, R., \& Jacobson, L. (1968). Pygmalion in the classroom. New York : Rinehart \& Winston.

Ross, L. (1977). The intuitive psychologist and his shortcomings : Distorsions in the attribution process. In L. Berkowitz (Ed.), Advances in experimental social psychology (Vol. 10, pp. 173- 220). New York : Academic Press.

Snyder, M. (1984). When belief creates reality. In L. Berkowitz (Ed.), Advances in experimental social psychology,Vol. 18. (pp. 248-306). New York : Academie Press.

Snyder, M., \& Swann, W. B. (1978a). Behavioral confirmation in social interaction : From social perception to social reality. Journal of Experimental Social Psychology, 14, 148-162.

Snyder, M., \& Swann, W. B. (1978b).Hypothesis-testing processes in social interaction. Journal of Personality and Social Psychology, 36, 1202-1212.

Thibaut, J. W., \& Kelley, H. H. (1959). The social psychology of groups. New York : Wiley. Thomdike, E. L. (1920). Intelligence and its uses. Harper's Magazine, 140, 227-235. Uleman, J. S., \& Bargh, J. A. (1989). Unintended thought. New York : Guilford Press.

Walker, R. E., \& Foley, J. M. (1973). Social Intelligence : Its history and measurement. Psychological Reports, 33, 451-459.

Weinstein, E. A. (1969). The development of interpersonal competence. In D. A. Goslin (Ed.), Handbook of socialization theory and research. Chicago : Rand McNally.

Wentzel, K. (1991a). Social competence at school : Relation between social responsability and academie achievement. Review of Educational Research, 61, 1, 1-24.

Wentzel, K. R. (1991b). Relations between social competence and academic achievement in early adolescence. Child Development, 62, 1066-1078.

Wentzel, K. (1993). Does being good make the grade ? Social behavior and academie competence in middle school. Journal of Educational Psychology, 85, 2, 357-364.

Wentzel, K. (1998). Social relationship and motivation in middle school : The raie of parents, teachers, and peers. Journal of Educational Psychology, 90, 2, 202-209.

Wentzel, K. R., \& Asher, S. R. (1995). The academie lives of neglected, rejected, popular, and controversial children. Child Development, 66, 754-763.

Wentzel, K. R., \& Cadwell, K. (1997). Friendship, peer acceptance, and group membership : Relations to academie achievement in middle school. Child Developrnent, 68, 6, 1198-1209. 
Word, C. O., Zanna, M. P., \& Cooper, J. (1974). The nonverbal mecliation of self-fulfilling

prophecies in interracial interaction. Journal of Experimental Social Psychology, 10, 109-120. Yzerbyt, V., \& Schadron, G. (1996). Connaître et juger autrui. Grenoble : Presses universitaires.

\section{NOTES}

1. À ce propos, le statut du toucher ressemble fort à celui du contact oeil-oeil: la fréquence et la durée du contact oeil-œil est à la fois un indicateur et un déterminant de la sympathie interpersonnelle.

2. Ces auteurs mettent en doute l'idée classique d'une dimension évaluative générale employé qu'il est « travailleur » peut signifier une valeur d'utilité positive mais pas nécessairement une valeur affective positive). Outre l'intérêt essentiel d'une telle approche en ce qu'elle souligne le rôle distinctif des utilités sociales (sociétales/ générales, organisationelles/locales, personnelles/spécifiques) et donc du contexte social dans les jugements de valeur, il n'en reste pas moins que (a) on peut envisager que les valeurs affectives et d'utilité puissent interagir entre elles, de sorte que, le jugement d'utilité puisse affecter celui de sympathie ou que le sentiment de sympathie puisse affecter celui d'utilité et que (b) même si l'on admet l'indépendance des deux dimensions au plan cognitif, la valeur affective reste un déterminant des comportements de l'évaluateur à l'égard de la cible et donc des comportements de la cible (y compris, peut-être, de ses comportements d'utilité) ce qui justifie que l'on accorde ici une attention particulière à la dimension affective des attitudes et des jugements de valeur interpersonnels.dans les descriptions d'autrui. Ils proposent de distinguer deux composantes essentielles de la valeur : la valeur affective et la valeur d'utilité de sorte que dire d'un employé qu'il est " une âme sensible » peut signifier une valeur affective positive mais pas nécessairement une valeur d'utilité positive (et inversement dire d'un

\section{RÉSUMÉS}

Juger et estimer la valeur d'autrui est un acte éminemment social qui constitue une question complexe et fascinante pour les psychologues sociaux. L'objectif de cet article est de passer en revue de manière aussi synthétique que possible les contributions majeures des recherches en psychologie sociale au problème du jugement social, afin de dégager des pistes de réflexion et d'action pour le professionnel, essentiellement dans le domaine scolaire. Nous montrons, dans un premier temps, en quoi les psychologues sociaux mettent en garde l'évaluateur contre les nombreux biais socio-cognitifs et socio-affectifs qui affectent ses jugements d'autrui. Nous suggérons, dans un second temps, que bien que les psychologues sociaux se soient essentiellement penchés sur la source du jugement, il est utile de prendre en compte la cible du jugement et l'interaction entre la cible et la source. À ce propos, la notion de compétence sociale nous semble essentielle. Elle ouvre une voie vers une meilleure compréhension des processus du jugement social, notamment dans le domaine scolaire. 
Judging and evaluating other people's value is truly a social act, which constitutes a complex and fascinating issue for social psychologists. The aim of this article is to review, as concisely as possible, the major contributions of social psychological research to the issue of social judgement. Thus, food for thought and action are provided to the professional, primarily in the domain of education. We will first show how social psychologists warn assessors against the numerous social cognitive and social affective biases that affect their judgements of others. Then, we will suggest that even if social psychologists have mainly focused their studies upon the on gin of judgements, it is worth taking into account the target of the judgernent as well as well as the interaction between the target and the origin. In this regard, die concept of social competence seems essential to us. It opens new ways towards a better understanding of social judgement processes in die domain of education and beyond.

\section{INDEX}

Mots-clés : Psychologie sociale, cognition sociale, jugement social, biais, attraction interpersonnelle, compétence sociale, éducation

Keywords : Social psychology, social cognition, social judgement, biases, interpersonal attraction, social competence, and education

\section{AUTEURS}

\section{ÉRIC DÉPRET}

est Maître de Conférences en Psychologie Sociale à l'Université Pierre Mendès France de Grenoble, membre de l'équipe de recherche du Laboratoire de Psychologie Sociale GrenobleChambéry (LPS). Contact : Université Pierre Mendes France, U.F.R. S.H.S., B.P. 47X, 38040 Grenoble Cedex 9, France. Courriel : Fric.Depret@upmf-grenoble.fr

\section{LAURENCE FILISETTI}

est Doctorante en Psychologie Sociale à l'Université Pierre Mendès France de Grenoble, membre de l'équipe de recherche du Laboratoire de Psychologie Sociale Grenoble-Chambéry (LPS). 